\title{
Description of Alvania rominae n. sp. from the Mediterranean Sea (Mollusca Gastropoda Rissoidae)
}

\author{
Bruno Amati ${ }^{*}$, Daniele Trono² \& Marco Oliverio ${ }^{3}$ \\ ${ }^{1}$ Largo Giuseppe Veratti 37/D, 00146 Roma, Italy; e-mail: bruno_amati@yahoo.it \\ ${ }^{2}$ Via E. Menga 14, 73043 Copertino, Lecce, Italy; e-mail: danieletrono@virgilio.it \\ ${ }^{3}$ Dipartimento di Biologia e Biotecnologie ‘Charles Darwin', Sapienza Università di Roma, Viale dell'Università 32, I-00185 Roma, \\ Italy; e-mail: marco.oliverio@uniroma1.it \\ *Corresponding author
}

\begin{abstract}
A new Mediterranean species of the genus Alvania Risso, 1826 (Gastropoda Rissoidae) is described: $A$. rominae n. sp. All examined specimens are from the Ionian Sea. It is compared with the most similar congeners from the Mediterranean Sea: A. lineata Risso, 1826 and $A$. settepassii Amati et Nofroni, 1985, with which it is sympatric, and A. schwartziana Brusina, 1866, A. elisae Margelli, 2001, A. bartolinorum Amati et Smriglio, 2019 and A. zaraensis Amati et Appolloni, 2019.
\end{abstract}

KEY WORDS Gastropoda; Rissoidae; Alvania; new species; Mediterranean Sea, taxonomy.

Received 30.05.2020; accepted 20.06.2020; published online 26.06.2020

\section{INTRODUCTION}

The genus Alvania Risso, 1826 (Gastropoda, Rissoidae) includes a group of small marine species living from the lower intertidal to bathyal depths, mostly on the continental shelf, where they are often associated with algal facies (e.g. Tringali, 2001; Gofas \& Oliver, 2011; Amati, 2012), with scarce representatives in deeper waters down to $4700 \mathrm{~m}$ depth (Bouchet \& Warén, 1993). They feed on diatoms, dinoflagellates, and detritus, with the deep-sea species being presumably selective deposit feeders (Ponder, 1985). The genus, as currently conceived, includes about 250 species (MolluscaBase, 2020) and is distributed almost worldwide (northeastern Atlantic and Mediterranean Sea, northwestern Atlantic, Caribbean, eastern Pacific, Indo-West Pacific, temperate Australia and South Africa: Ponder, 1985). This is one of the nominally most diverse genera of gastropods and is particu- larly species-rich in the Mediterranean Sea, with over 70 recognized species. In the Mediterranean Sea, Alvania comprises species of small to medium size for the family (from $1 \mathrm{~mm}$ length of $A$. maximilicutiani Scuderi, 2014, to $7 \mathrm{~mm}$ length of $A$. mamillata Risso, 1826: Scuderi, 2014; Amati et al., 2017). The shell is ovate-conical, generally with axial and spiral elements forming a delicate to robust clathrate sculpture, a continuous peristome, and a variable colouration.

The genus Alvania includes several species groups defined morphologically, as for instance the complexes of A. lineata Risso, 1826 (Amati et al., 2019), A. dictyophora (Philippi, 1844) (Amati \& Smriglio, 2016), A. subcrenulata (Bucquoy, Dautzenberg et Dollfuss, 1884) (Amati, 2014), A. cimex (Linnaeus, 1758) (Amati et al., 2017), A. rudis (Philippi, 1844) (Amati et al., 1987) and A. scabra (Philippi, 1844) (Amati et al., 2020). Among them, the $A$. lineata-complex includes some 23 recogni- 
sed extant species (Amati et al., 2019), all with non-planktotrophic development (and paucispiral protoconch), except for A. discors (Allan, 1818) which has a planktotrophic larval stage (and multispiral protoconch). For a description and a recent revision of the A. lineata group we refer to Amati et al. (2019). Traditionally, the taxonomy of Mediterranean rissoids has been based almost exclusively on shell characters, whose puzzling variation has often caused uncertainty about the status of some of these taxa. Admittedly, studies integrating multiple sources of information (teleoconch, protoconch, soft parts morphology and colour pattern, radula, and particularly, genetics) would certainly be crucial in assessing their taxonomy. However, in most cases, the availability of large samples and the comparative study of type material provide enough important data to improve current taxonomic knowledge.

Several specimens of the A. lineata complex from the Ionian Sea are morphologically distinct from all other known members and are described herein as a new species: A. rominae $\mathrm{n}$. sp.

\section{MATERIAL AND METHODS}

The samples studied here are stored in public and private collections, as detailed below (see Abbreviations and acronyms). Photographs have been taken with a Sony Cyber-Shot digital camera mounted on a Kyowa KBS stereomicroscope, edited with the Combine- $Z$ software (Hadley, 2006). The adopted rissoid taxonomy is after MolluscaBase (MolluscaBase, 2020: accessed through the World Register of Marine Species, WoRMS). Measurements were taken on a random sample of 15 specimens (full-grown intact adults) and are reported in Table 1.

ABBREVIATIONS AND ACRONYMS. AF: Angelo Fiorita collection (Porto Cesareo, Lecce, Italy); AR: Alessandro Raveggi collection (Firenze, Italy); AV: Angelo Vannozzi collection (Roma, Italy); BA: Bruno Amati collection (Roma, Italy); CS-PM: Carlo Smriglio-Paolo Mariottini collection (Roma, Italy); DS: Danilo Scuderi collection (Scordia, Catania, Italy); DT: Daniele Trono collection (Copertino, Lecce, Italy); EQ: Ermanno Quaggiotto collection (Longare, Vicenza, Italy); IN: Italo Nofroni collection (Roma, Italy); lv: live collected specimens; MCZR: Museo Civico di Zoologia (Roma, Italy); MNHN: National Museum of Natural History (Paris, France); MO: Marco Oliverio collection (Roma, Italy); SB-MS: Stefano Bartolini-Maria Scaperrotta collection (Firenze, Italy); sh: empty shell(s); WR: Walter Renda collection (Amantea, Cosenza, Italy).

\section{RESULTS}

\section{Systematics}

Classis GASTROPODA Cuvier, 1795

Subclassis CAENOGASTROPODA Cox, 1960

Superfamilia RISSOOIDEA Gray, 1847

Familia RISSOIDAE Gray, 1847

Genus Alvania Risso, 1826

Type-species: Alvania europea Risso, 1826: 142, pl. IX, fig. $116=$ Alvania cimex (Linnaeus, 1758) (Turbo), by subsequent designation Nevill, 1885: 105.

Alvania rominae n. sp. (Figs. 1-22; Table 1) https://zoobank.org:act:421E1F98-B6BE-4BFC 870B-B8D1DB71B227

Type Material. Holotype MNHN-IM-200035744, H 3.5 mm, W 2.05 mm (Figs. 2-4, 14, 15, 22); 11 paratypes (DT), 1 paratype MCZR-MTYPE00093/P; 1 paratype (BA), 1 paratype (MO), 1 paratype (AF); 1 paratype (WR); 1 paratype (AV); type locality: Gallipoli, Lecce $40^{\circ} 00^{\prime} 95^{\prime \prime} \mathrm{N}$ $17^{\circ} 55^{\prime} 32^{\prime \prime} \mathrm{E}, 58 \mathrm{~m}$ depth, Ionian Sea.

OTHER EXAMINED MATERIAL. Alvania rominae $\mathrm{n}$. sp. Italy: Otranto, Lecce $40^{\circ} 13^{\prime} 85^{\prime} \mathrm{N} 18^{\circ} 51^{\prime} 35^{\prime \prime} \mathrm{E}$, 35 m depth, VIII.2004, 10 sh (DT); S. Isidoro, Lecce $40^{\circ} 08^{\prime} 28^{\prime \prime} \mathrm{N} 17^{\circ} 49^{\prime} 13^{\prime \prime} \mathrm{E}, 53 \mathrm{~m}$ depth, VIII.2008, 8 sh (DT); S. Isidoro, Lecce $40^{\circ} 08^{\prime} 28^{\prime \prime} \mathrm{N}$ 17049'13"E, 53 m depth, 29.VII.2018, 2 sh (DT); Porto Cesareo, Lecce $40^{\circ} 11^{\prime} 48^{\prime \prime} \mathrm{N} 17^{\circ} 47^{\prime} 08^{\prime} \mathrm{E}, 40$ m depth, III.2019, 2 sh (DT).

Alvania elisae Margelli, 2001 (Fig. 28): Type material: 1 paratype, Capraia Island, $2-15 \mathrm{~m}$ depth (CS-PM, ex M.A. Fontana Angioy coll., Rome); Italy: Capri Island, unspecified depth, 7 sh (MCZR-M-30060); Elba Island, Capo di Fonza, $25 \mathrm{~m}$ depth, $80 \mathrm{sh}$ (EQ), $7 \mathrm{sh}$ (BA); Elba Island, Capo di Stella, 20 m depth, 13 sh (EQ); Elba Is- 
land, 3 sh (MO); Capraia Island, P.ta della Fica, 24 $\mathrm{m}$ depth, 3 sh (DT).

Alvania lineata Risso, 1826 (Figs. 29-51): Spain: Fuengirola, $30 \mathrm{~m}$ depth, VIII.1985, $45 \mathrm{sh}$ (BA); Malaga, 5-10 m depth 11.XI.2009, $30 \mathrm{sh}$ (BA); Tunisia: Gulf of Gabès, Kerkennah, 1974, 24 sh (BA); Gulf of Gabès, Kerkennah, loc. Sidi youssef, VII.2015, 200 sh (BA); Corsica, Barcaggio, 20 m depth, 4 sh (DT); Sardinia: San Teodoro, 1 sh (BA); Cala Gonone, Dorgali, $1 \mathrm{~m}$ depth, VIII.2010, 25 lv (BA), 2-4 m depth, VIII.2010, 61 sh (BA); Maddalena Island, 1989, $24 \mathrm{~m}$ depth, $1 \mathrm{sh}$ (BA); Golfo Aranci, 1985, 3 sh (BA); Oristano, VIII.1978, 4 sh (BA); Sicily: Salina Island, 35 m depth, VII.2002, 500 sh (BA); Levanzo Island, Punta Altarella $31 \mathrm{~m}$ depth, 3.VI.1991, 6 sh (BA); Marettimo Island, Egadi, 19 sh (BA); Magnisi, Beached, legit Oberling (13.III.1970), 35 sh, (BA); Portopalo di Capo Passero, 1 sh (BA); Lampedusa Island, Capo Grecale, VII.2009 $50 \mathrm{~m}$ depth $10 \mathrm{sh}$ (BA); Cannizzaro, Catania, 35-43 m depth, $61 \mathrm{sh}$ (BA); Ognina, Siracusa, 13 sh (BA) 1984, washing algae, $24 \mathrm{lv}$ (BA); Lampedusa Island, $30 \mathrm{~m}$ depth, 53 sh (BA); Lampedusa Island, Cala Calandra 30 m depth, 30.IV.1991, 46 sh (BA); Vendicari Island, 28 m depth, 23 sh (BA); Ragusa, Roventa Shoal, 27 m depth, VI.1989, 8 sh (BA); Marettimo Island, Egadi I., 10 sh (BA); Linosa Island, P.ta Calcarelle, $36 \mathrm{~m}$ depth, $3 \mathrm{sh}$ (DT); Stretto di Messina, $19 \mathrm{~m}$ depth, 5 sh (DT); Malta: Malta Island, beached bioclastic sand, 1 sh (BA); Italy: Giglio Island 27-36 $\mathrm{m}$ depth, $45 \mathrm{sh}$ (BA); Capraia Island 200-280 m depth, 1979, 1 sh (BA), 140-180 m depth, 1978, 16 sh (BA); Capraia Island, P.ta della Fica, $24 \mathrm{~m}$ depth, $4 \mathrm{sh}$ (DT); Secca di Torre Flavia $26 \mathrm{~m}$ depth, 1986, 64 sh (BA); Secche di Tor Paterno, Rome, $30 \mathrm{~m}$ depth, 1.X.1988, $4 \mathrm{sh}$ (BA), $45 \mathrm{~m}$ depth, $18 \mathrm{sh}$ (BA); Santo Stefano Island, $40 \mathrm{~m}$ depth, $14 \mathrm{sh}$ (BA); Ventotene Island, 1979, 25 sh (BA); Torre Astura, Rome, 1977, 1 sh (BA); San Felice Circeo, Latina, $30 \mathrm{~m}$ depth, $10 \mathrm{sh}$ (BA), 4-6 m depth, $43 \mathrm{sh}$ (BA-MO), 20 m depth, 8 sh (DT); Pozzano, Reggio Calabria, 40 m depth, VIII.1986, 1 sh (BA); Scilla, $41 \mathrm{~m}$ depth, 16.XII.2006, legit Marconcini, $48 \mathrm{sh}$ (IN); Gallipoli, Lecce, 2,5 m depth, $1 \mathrm{sh}$ (DT); Nardò, Lecce, loc. S. Caterina, $30 \mathrm{~m}$ depth in the cave, 3 sh (DT), 18-23 m depth, 12 sh (DT); Nardò, Lecce, loc. Torre Inserraglio, $20 \mathrm{~m}$ depth, $13 \mathrm{sh}$ (IN), $20 \mathrm{~m}$ depth, $28 \mathrm{sh}$ (DT); Porto Cesareo, Lecce, tide pools, VIII.1976, 11 sh (IN), VIII.1988,
20 sh (IN); Secca di Pellaro, $25 \mathrm{~m}$ depth, $18 \mathrm{sh}$, (DT); Castro, Lecce, $23 \mathrm{~m}$ depth, $10 \mathrm{sh}$ (DT); Croatia: Umag, beached bioclastic sand, 73 sh (BA); Umag, 1978, 7 sh (BA); San Lorenzo, 5 sh (BA); Slovenija: Portoroz, beached bioclastic sand, 1 sh (BA); Greece: undefined locality, 29 sh (BA); Crete, 34 sh (MCZR-M-30127); Crete, beached bioclastic sand, 5 sh (BA); Mediterranean Sea, undefined localities, 75 sh (MCZR-M-22149).

Alvania settepassii Amati et Nofroni, 1985 (Figs. 23, 24 ): Type material: holotype (MCZRM-TYPE00004/H) and 42 paratypes, Secche di Tor Paterno, $45 \mathrm{~m}$ depth (BA); 69 paratypes Civitavecchia, 25-30 m depth, (BA); Italy: Giglio Island, 27-30 m depth, $16 \mathrm{sh}$ (BA); S. Stefano Island, 40 $\mathrm{m}$ depth, 1 sh (BA); Torvaldaliga, Civitavecchia, 20-22 m depth, 250 sh (CS-PM); Secche di Torre Flavia, Ladispoli $26 \mathrm{~m}$ depth 1986, 3 sh (BA); Torre Astura, beached, 3 sh (BA); Nettuno, beached, 1982, 1 sh (BA); San Felice Circeo, Latina, 4-6 m depth, 69 sh (BA-MO), $20 \mathrm{~m}$ depth, 4 sh (DT); Nardò, Lecce, loc. S. Caterina, 18-23 $\mathrm{m}$ depth, 6 sh (DT); Nardò, Lecce, loc. Torre Inserraglio, $20 \mathrm{~m}$ depth, $13 \mathrm{sh}$ (IN), 20-22 $\mathrm{m}$ depth, $44 \mathrm{sh}$ (DT); Secca di Pellaro, $25 \mathrm{~m}$ depth, $4 \mathrm{sh}$ (DT); Gallipoli, Lecce, $30 \mathrm{~m}$ depth, 4 sh (DT); Castro, Lecce, $23 \mathrm{~m}$ depth, 11 sh (DT); Porto Cesareo, Lecce, 30 m depth, 4 sh (DT); Nardò, Lecce, loc. Torre Uluzzo, 29 -34 m depth, 2 sh (DT); Sicily: Vendicari Island, $28 \mathrm{~m}$ depth, $1 \mathrm{sh}$ (BA); Croatia: Lastovo Island $38 \mathrm{~m}$ depth, $21 \mathrm{sh}$ (BA); Greece: Crete, $960 \mathrm{~m}$ depth, 3 sh (AP).

Alvania schwartziana Brusina, 1866 (Fig. 25): Type material: 4 syntypes, Zadar, Croatia (MCZRM-22326, Monterosato coll. ex Brusina). Croatia: Zadar and Pag, 8 sh (BA); Starigrad Paklenica, Zadar, $6 \mathrm{~m}$ depth, $29 \mathrm{sh}$ (EQ); Dalmatia, $40 \mathrm{~m}$ depth, 2 sh (DT); Italy: Chioggia, Venice, $20 \mathrm{~m}$ depth, 2 sh (EQ).

Alvania bartolinorum Amati et Smriglio, 2019 (Fig. 26): Type material: holotype, (MNHN-IM2000-27706) and paratypes (BA, CS-PM, SB-MS, AR, EQ), Krk Island, Croatia. Croatia: Krk Island, $54 \mathrm{~m}$ depth, IX.2008, 4 sh (AR), 252 sh (SB-MS), 16 sh (EQ); Krk Island, Giapni Potok, 54 m depth, $23 \mathrm{sh}$ (AR).

Alvania zaraensis Amati et Appolloni, 2019 (Fig. 27). Type material: holotype, (MCZR-M22152/H) and 14 paratypes, Zadar, Croatia (MCZR$\mathrm{M}-22152 / \mathrm{P})$. 
DiAGNOSIS. Medium sized rissoid height 2.9-3.6 $\mathrm{mm}$ with axial sculpture of $8-12$ ribs interrupted at the periphery on the last whorl; spiral sculpture of 9-11 non-equidistant spiral cords on the last whorl, of which 5 above the aperture and with variable chromatic pattern.

Description of the Holotype. Shell (Figs. 2$4 ; 14,15,22)$ solid, oval-conical, of medium size for the genus, height $3.5 \mathrm{~mm}$, width $2.05 \mathrm{~mm}, \mathrm{H} / \mathrm{W}$ ratio 1.707. Protoconch (Fig. 15) paucispiral, globose, of 1.3 whorls, height $0.35 \mathrm{~mm}$, nucleus diameter $0.15 \mathrm{~mm}$, first half whorl diameter $0.25 \mathrm{~mm}$, maximum diameter $0.40 \mathrm{~mm}$; sculpture of a dozen slightly wavy spiral threads, partially anastomized. Teleoconch of 4.25 slightly convex whorls, suture impressed, especially in the first whorls. Axial sculpture of 9 ribs interrupted at the periphery on the last whorl, as wide as the interspaces or slightly narrower; always orthocline on the first two whorls, from slightly opisthocline to slightly prosocline on the others. Spiral sculpture of 11 non-equidistant spiral cords on the last whorl, of which 5 above the aperture, one sutural, and 5 more spaced, on the base; two cords (III and V) starting immediately after protoconch/teleoconch boundary, followed by cord I, IV and finally II. Basal cords decreasing in size and strength, last one barely noticeable. Microsculpture of spiral minute threads and weak growth lines. Umbilical fold absent. Aperture large, pyriform, height $1.5 \mathrm{~mm}, \mathrm{H} / \mathrm{Ha}$ ratio 2.333 . Outer lip sharp, with large prosocline varix, without fine elongated internal teeth. Colour yellow-orange background, with darker spiral cords. Base, internal aperture and varix whitish. Operculum and soft parts unknown.

VARIABILITY. Paratypes variation (see also Table 1): shell height $2.9-3.6 \mathrm{~mm}$, width $1.75-2.1 \mathrm{~mm}$, $\mathrm{H} / \mathrm{W}$ ratio 1.611-1.846. Protoconch (Figs. 17, 18, 20) paucispiral, globose, of $1.25-1.5$ whorls, height $0.325-0.40$, nucleus diameter $0.125-0.150$, first half whorl diameter $0.225-0.275 \mathrm{~mm}$, maximum diameter $0.387-0.425 \mathrm{~mm}$. Teleoconch whorls 3.8-4.25; axial sculpture of 8-12 ribs; spiral cords on the last whorl 9-11, of which 5 above the aperture, one sutural, and 3-5 more spaced, on the base. Aperture height $1.25-1.6 \mathrm{~mm}, \mathrm{H} / \mathrm{Ha}$ ratio 2.250 2.560. Outer lip sharp, with large prosocline varix, often with 7-8 fine elongated internal teeth. Colour whitish-yellowish to brown-beige background with pinkish hue; usually first whorls darker brown-orange, pink-violet aperture, and darker spiral cords; brown or whitish-yellowish monochrome shells not rare.

DisTRIBUTION AND BIOLOGY. Examined material from the Ionian Sea (Italy): Gallipoli, Porto Cesareo, S. Isidoro, Otranto. Empty shells in $35-58 \mathrm{~m}$ depth. (Fig. 1). Sympatric in the Ionian Sea with $A$. lineata and A. settepassii.

Etymology. After Mrs. Romina Leo, wife of one of the authors (Daniele Trono).

REMARKS. The examined materials showed a negligible morphological variation, except for the variable chromatic pattern of the shell, with monochromatic brown or whitish-yellowish shells not rare. Almost half of the specimens examined had some elongated teeth inside the outer lip. Other species are characterised by an inconsistent presence of teeth, as e.g., A. mamillata Risso, 1826, A. dictyophora (Philippi, 1844), A. hallgassi Amati et Oliverio, 1985 and A. desabatae Amati et Smriglio, 2016 (Amati et al., 2020). Micali et al. (2005: 23, fig. 2) figured as Alvania sp. a shell from Tremiti Islands similar to $A$. rominae n. sp. but more likely belonging to $A$. bartolinorum. That specimen is stated to be conspecific with samples listed by Chiarelli \& Micali (2003: 16) from Vela Luka (Croatia). Instead, the two specimens from

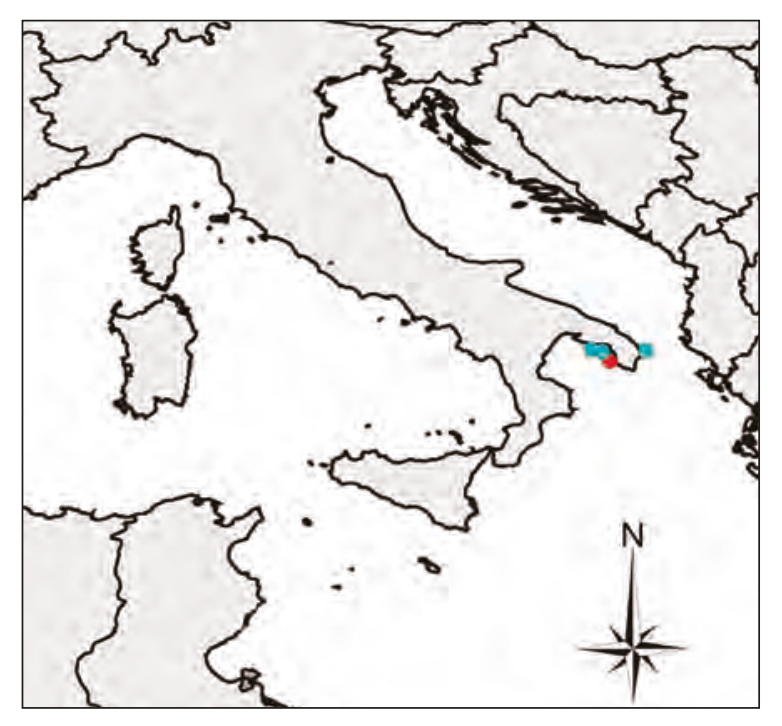

Figure 1. Distribution of Alvania rominae n. sp., based on the material examined (red 'type material' and light-blue dots 'other examined material'). 


\begin{tabular}{|c|c|c|c|c|c|c|c|c|c|c|c|c|c|c|c|c|c|c|}
\hline Teleoconch & 1 & 2 & 3 & 4 & 5 & 6 & 7 & 8 & 9 & 10 & 11 & 12 & 13 & 14 & 15 & Min-max & Mean & Std \\
\hline Height & 3.25 & 290 & 3.50 & 3.55 & 3.60 & 3.55 & 3 & 3.20 & 335 & 3,25 & 3 & 3.02 & 320 & 3 & 305 & $290-3.60$ & 3.228 & 0.235 \\
\hline Widht & 1.95 & 1.80 & 2.05 & 2 & 1.95 & 2.10 & 1.85 & 1.85 & 1.90 & 1.85 & 1.75 & 1.80 & 1.80 & 1.75 & 1.75 & $1.75 \cdot 2.10$ & 1.877 & 0.112 \\
\hline Aperture height & 1.4 & 1.25 & 1.5 & 1.45 & 1.6 & 1.55 & 1.25 & 1.25 & 1.35 & 1,35 & 1.25 & 1,3 & 1,3 & 1.25 & 1.27 & $1.25 \cdot 1,60$ & 1.355 & 0.119 \\
\hline Heightwidth ratio & 1.666 & 1.611 & 1.707 & 1.775 & 1.846 & 1.690 & 1.621 & 1.729 & 1.763 & 1.756 & 1.714 & 1.677 & 1.777 & 1.714 & 1.742 & $1.611-1.846$ & 1.719 & 0,062 \\
\hline Height/aperture height ratio & 2.321 & 2.320 & 2.333 & 2.448 & 2.250 & 2.290 & 2.400 & 2.560 & 2,481 & 2407 & 2400 & 2.323 & 2.461 & 2.400 & 2.401 & $2250-2.560$ & 2.386 & 0,082 \\
\hline \begin{tabular}{|l|}
$N^{\circ}$ of whorls \\
\end{tabular} & 42 & 4 & 4.25 & 4.2 & 4 & 4 & 4.1 & 4.1 & 4.25 & 4.2 & 4.1 & 3.8 & 4.1 & 4 & 4 & $3.8-4.25$ & 4.087 & 0.123 \\
\hline $\begin{array}{l}\mathrm{N}^{\circ} \text { axial ribs on last whorls } \\
\text { (varix excluded) }\end{array}$ & 10 & 11 & 9 & 9 & 10 & 8 & 9 & 10 & 10 & 9 & 10 & 9 & 9 & II & 12 & $8-12$ & 9.733 & 1.033 \\
\hline$N^{\circ}$ spiral cords on last whorls & II & II & 11 & 9 & II & 10 & 10 & 9 & 11 & 10 & 9 & 10 & 10 & 9 & 10 & $9-11$ & 10.066 & 0.884 \\
\hline $\begin{array}{l}N^{\circ} \text { spiral cords on the base } \\
\text { (excluding sutural cord) }\end{array}$ & 5 & 5 & 5 & 3 & 5 & 4 & 4 & 3 & 5 & 4 & 3 & 4 & 4 & 3 & 4 & 3.5 & 4.066 & 0.737 \\
\hline $\begin{array}{l}\text { No number of denticles inside } \\
\text { the aperture }\end{array}$ & 8 & no & no & 7 & 8 & 8 & no & 8 & no & no & 7 & 7 & no & ino & 8 & no/7.8 & 7.625 & 0.518 \\
\hline Protoconch & 1 & 2 & 3 & 4 & 5 & 6 & 7 & 8 & 9 & 10 & 11 & 12 & 13 & 14 & 15 & Min-max & Meam & Sid \\
\hline Height & 0.350 & 0.375 & 0.350 & 0.400 & 0.400 & 0.375 & 0375 & 0,350 & 0.375 & 0.375 & 0.325 & 0350 & 0.350 & 0.350 & 01,337 & $0.325-0.400$ & 0.362 & 0.022 \\
\hline Diameter of nucleus & 0.125 & 0.125 & 0.150 & 0.150 & 0.150 & 0.150 & 0.137 & 0.150 & 0.137 & 0.150 & 0.125 & 0.125 & 0.137 & 0.150 & 0.137 & $0.125-0.150$ & 0.140 & 0.011 \\
\hline \begin{tabular}{|l|l|} 
Diameter of first half whorl \\
\end{tabular} & 0.250 & 0.250 & 0.250 & 0.275 & 0.275 & 0.250 & 0.250 & 0.262 & 0.250 & 0275 & 0.225 & 0.250 & 0.250 & 0.275 & 0.250 & $0.225-0.275$ & 0.256 & 0.014 \\
\hline Maximum diameter & 0.400 & 0.425 & 0.400 & 0.400 & 0.412 & 0.425 & 0.400 & 0.425 & 0.400 & 0.412 & 0.400 & 0.425 & 0.400 & 0.425 & 0.387 & $0.387-0.425$ & 0.409 & 0.013 \\
\hline$N^{\circ}$ of whorls & 13 & 1.3 & 13 & 14 & 14 & 14 & 1.35 & 13 & 1.4 & 1.25 & 1.25 & 1.25 & 15 & 1.25 & 15 & 125.15 & 1.343 & 0086 \\
\hline
\end{tabular}

Table 1. Measurements of teleoconch and protoconch of Alvania rominae n. sp. in mm, with range, mean and standard deviation. $1=\mathrm{S}$. Isidoro $53 \mathrm{~m}$ depth $(\mathrm{SEM}) ; 2=$ Otranto $35 \mathrm{~m}$ depth $(\mathrm{SEM}) ; 3=$ holotype, Gallipoli $58 \mathrm{~m}$ depth $(\mathrm{SEM}) ; 4=$ Gallipoli $-58 \mathrm{~m} ; 5=$ Gallipoli $58 \mathrm{~m}$ depth; $6=$ Gallipoli $58 \mathrm{~m}$ depth; $7=$ Gallipoli $58 \mathrm{~m}$ depth; $8=$ Otranto $35 \mathrm{~m}$ depth; $9=$ Otranto $35 \mathrm{~m}$ depth; $10=$ Otranto $35 \mathrm{~m}$ depth; 11= P. Cesareo $40 \mathrm{~m}$ depth; 12= P. Cesareo $40 \mathrm{~m}$ depth; $13=\mathrm{S}$. Isidoro 53 $\mathrm{m}$ depth; $14=\mathrm{S}$. Isidoro $-53 \mathrm{~m} ; 15=\mathrm{S}$. Isidoro $53 \mathrm{~m}$ depth.

Otranto illustrated by Scuderi \& Terlizzi (2012), and indicated as conspecifics with the specimens of the Tremiti Islands figured by Micali et al. (2005), are not this species and are rather to be referred to one of the morphs of $A$. lineata (see e.g. the 4 spiral cords above the aperture vs 5 in $A$. rominae $\mathrm{n}$. $\mathrm{sp}$.).

A. rominae n. sp. differs from $A$. elisae by its fewer and narrower axial ribs on the last whorl (812 vs $15-16$ in $A$. elisae), the wider interspaces, the 5 spiral cords above the aperture vs 4 in $A$. elisae.

Alvania rominae n. sp. differs from $A$. lineata in its more obsolete spiral sculpture on the last whorl, the 5 spiral cords above the aperture (vs usually 4, rarely 5 in $A$. lineata), and for the axial ribs interrupted at the periphery: A. lineata, in its most common morphotypes, has a stronger sculpture, especially the spirals, with marked nodules at intersections, a thicker varix and the axial ribs reaching the base.

Alvania rominae n. sp. differs from $A$. settepassii in its variable chromatic pattern vs a rather constant one in A. settepassii, cream-orange to brown with darker axial interspaces; narrower axial ribs, the lack of a subsutural shoulder $v s$ a scalariform outline with subsutural shoulder in A. settepassii.

Alvania rominae n. sp. differs from $A$. schwartziana in its globose and more strongly sculptured protoconch, with a dozen slightly wavy spiral rows, partially anastomized vs a more acute protoconch with slightly convex whorls and with a more delicate sculpture (after comparison with four syntypes, from Zadar (Croatia), ex Brusina in the Monterosato collection MCZR-M-22326), its smaller protoconch (maximum diameter 0.387$0.425 \mathrm{~mm}$ vs $0.511 \mathrm{~mm}$ in $A$. schwartziana), the 5 spiral cords above the aperture vs 4 in A. schwartziana. Different chromatic pattern, monochrome brown in $A$. schwartziana.

Alvania rominae n. sp. differs from A. bartolinorum in its smaller protoconch (maximum diameter $0.387-0.425 \mathrm{~mm}$ vs $0.440-0.500 \mathrm{~mm}$ in $A$. bartolinorum), and its usually fewer axial ribs on the last whorl (8-12, mean $9.73[\mathrm{~N}=15]$ vs $12-18$, mean $14.25[\mathrm{~N}=25]$ in A. bartolinorum). The number of spiral cords on the last whorl above the aperture are always 5 in $A$. rominae n. sp. vs 4-6 in $A$. bartolinorum. Alvania rominae n. sp. seems to be on average smaller: H. 2.90-3.60 mm (mean 3.228 $\mathrm{mm}$ ) $[\mathrm{N}=15]$ vs H. $2.84-3.96 \mathrm{~mm}$ (mean $3.415 \mathrm{~mm}$ ) $[\mathrm{N}=25]$ in A. bartolinorum.

Alvania rominae n. sp. differs from $A$. zaraensis in its protoconch, larger (maximum diameter $0.387-0.425 \mathrm{~mm}$ vs $0.325-0.375 \mathrm{~mm}$ in A. zaraensis), and with strong sculpture vs devoid of a strong sculpture in A. zaraensis, in the 5 spiral cords above the aperture vs 4 in $A$. zaraensis, the umbilical chink absent vs umbilical chink very narrow in A. zaraensis. Double sculpture (axial and spiral) much more robust with greater thickness. 


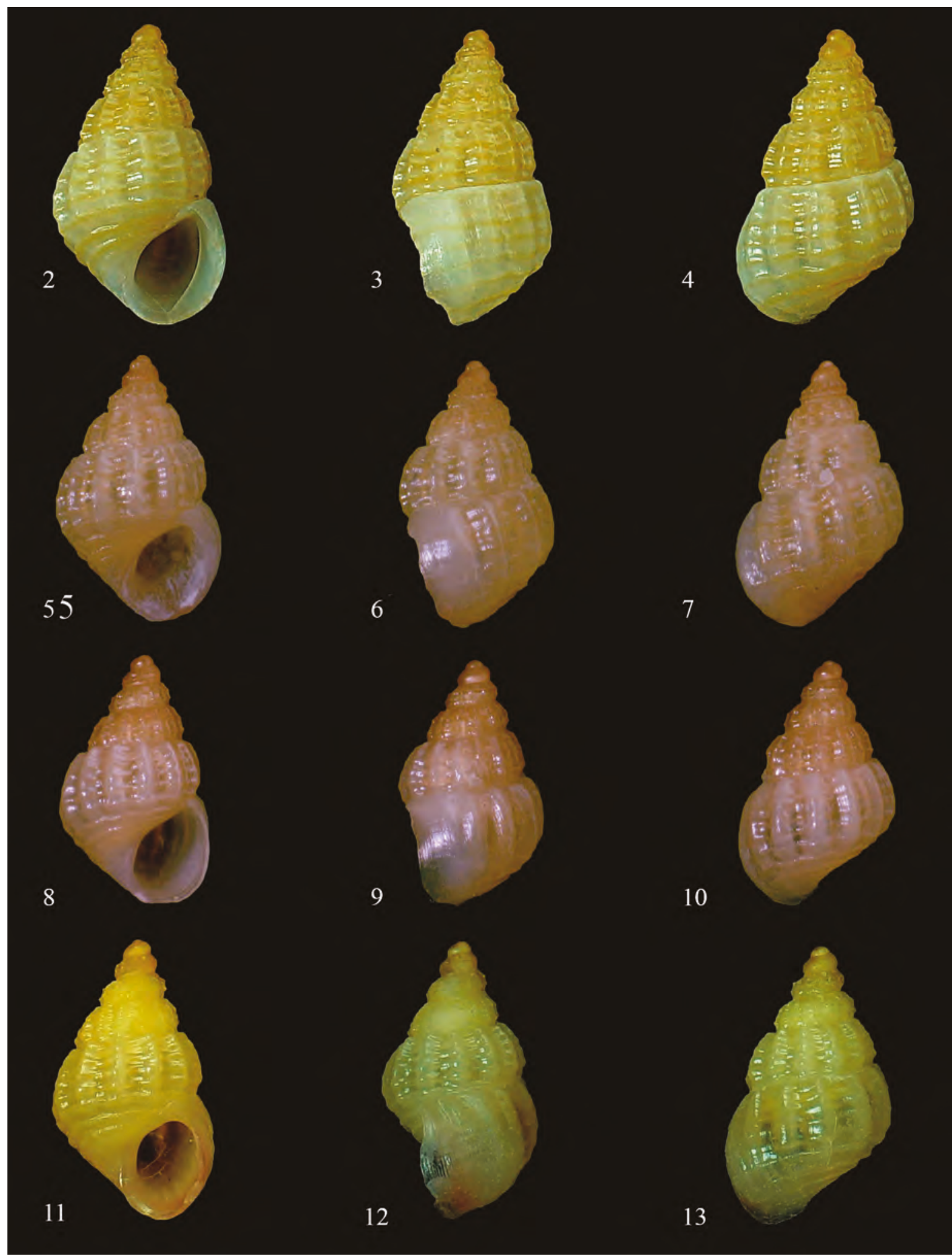

Figures 2-13. Alvania rominae n. sp. Figs. 2-4: holotype, Gallipoli (Italy) $58 \mathrm{~m}$ depth, height $3.5 \mathrm{~mm}$ (MNHN-IM-200035744). Figs. 5-7: paratype, Gallipoli (Italy) $58 \mathrm{~m}$ depth, height $3.2 \mathrm{~mm}$ (DT). Figs. 8-10: paratype, Otranto (Italy) $35 \mathrm{~m}$ depth, height $2.9 \mathrm{~mm}$ (DT). Figs. 11-13: paratype, S. Isidoro (Italy) $53 \mathrm{~m}$ depth, height $3.25 \mathrm{~mm}$ (DT). 


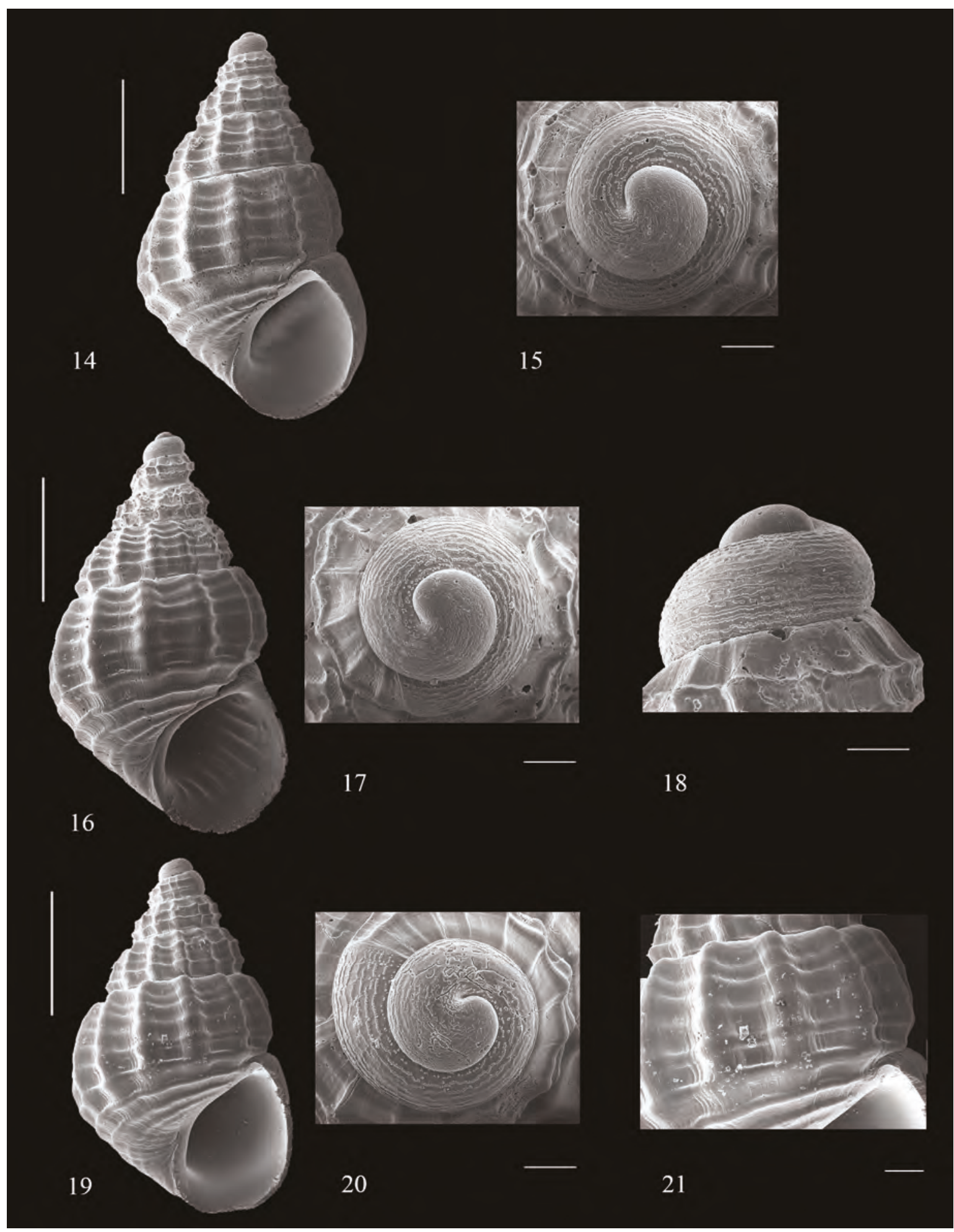

Figures 14-21. Alvania rominae n. sp. (SEM). Fig. 14-15. Holotype, Gallipoli (Italy) $58 \mathrm{~m}$ depth: shell (Fig. 14), detail of the protoconch microsculpture (MNHN-IM-2000-35744) (Fig. 15). Figs. 16-18. Paratype, S. Isidoro (Italy): shell (Fig. 16), detail of the protoconch microsculpture (DT) (Figs. 17, 18). Figs. 19-21. Paratype, Otranto (Italy): shell (Fig. 19), detail of the protoconch and teleoconch microsculpture respectively (DT) (Figs. 20, 21). Scale bars $=1 \mathrm{~mm}(14,16$ and $19),=100 \mu \mathrm{m}(15,17,18,20),=200 \mu \mathrm{m}(21)$. 


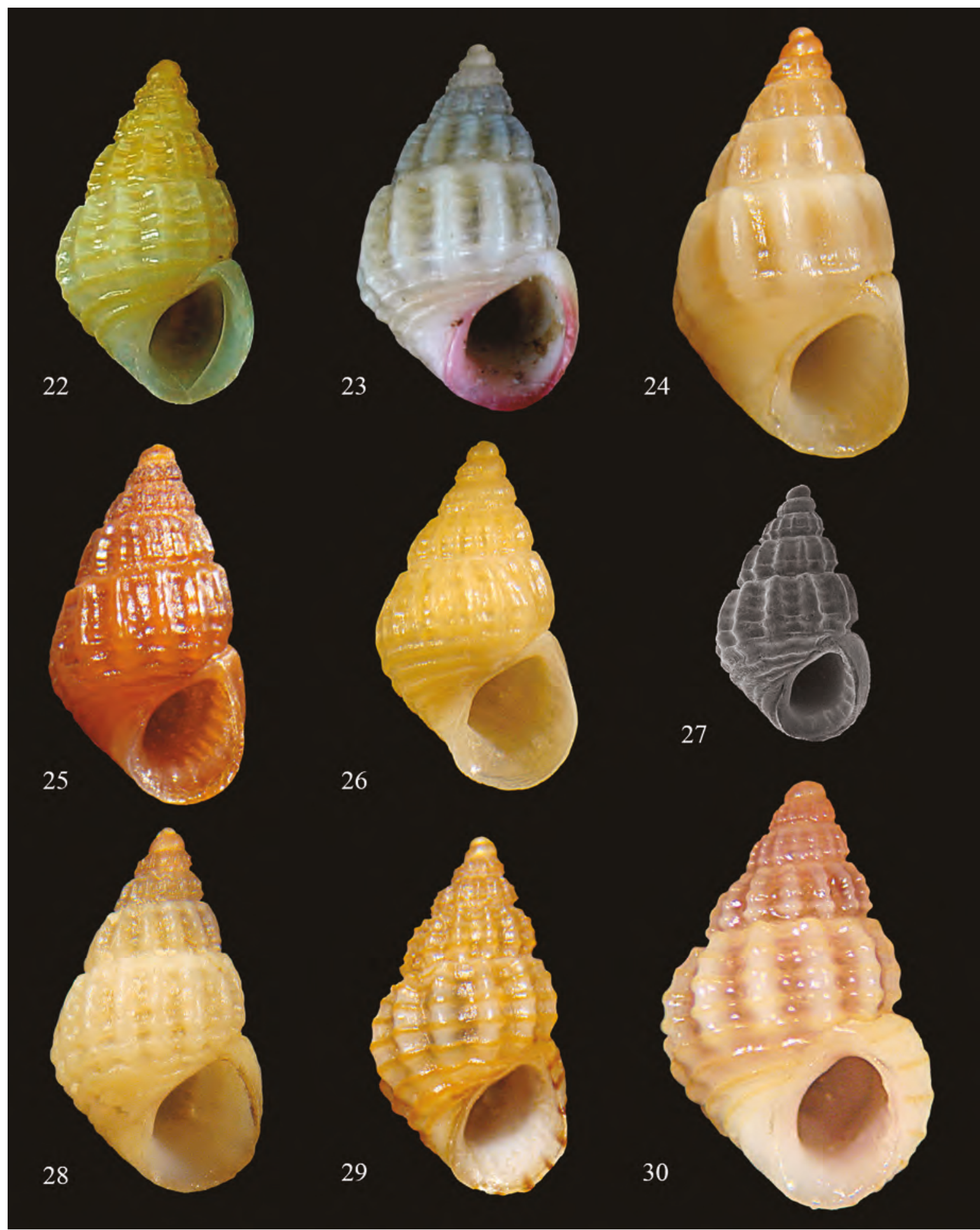

Figure 22. Alvania rominae n. sp. holotype, Gallipoli (Italy) $58 \mathrm{~m}$ depth, height $3.5 \mathrm{~mm}$ (MNHN-IM-2000-35744). Figure 23. Alvania settepassii, Porto Cesareo (Italy) $30 \mathrm{~m}$ depth, height $3.7 \mathrm{~mm}$ (DT). Figure 24. Alvania settepassii, paratype, Torrevaldaliga, Civitavecchia (Italy) 25-30 m depth, height $4.3 \mathrm{~mm}$ (BA). Figure 25. Alvania schwartziana, Pag (Croatia), height 3.6 $\mathrm{mm}$ (BA). Figure 26. Alvania bartolinorum, holotype, Krk Island (Croatia) $54 \mathrm{~m}$ depth, height $3.58 \mathrm{~mm}$ (MNHN-IM-200027706). Figure 27. Alvania zaraensis, holotype, Zadar (Croatia), height $2.6 \mathrm{~mm}$ (MCZR-M-22152/H). Figure 28. Alvania elisae, paratype, Capraia Island (Italy) 2-15 m depth, height $3.75 \mathrm{~mm}$ (CS-PM). Figure 29. Alvania lineata, Salina Island (Sicily) $35 \mathrm{~m}$ depth, height $3.65 \mathrm{~mm}$ (BA). Figure 30. Alvania lineata, Cala Gonone, Dorgali (Sardinia) $1 \mathrm{~m}$ depth, height $4.3 \mathrm{~mm}$ (BA). 

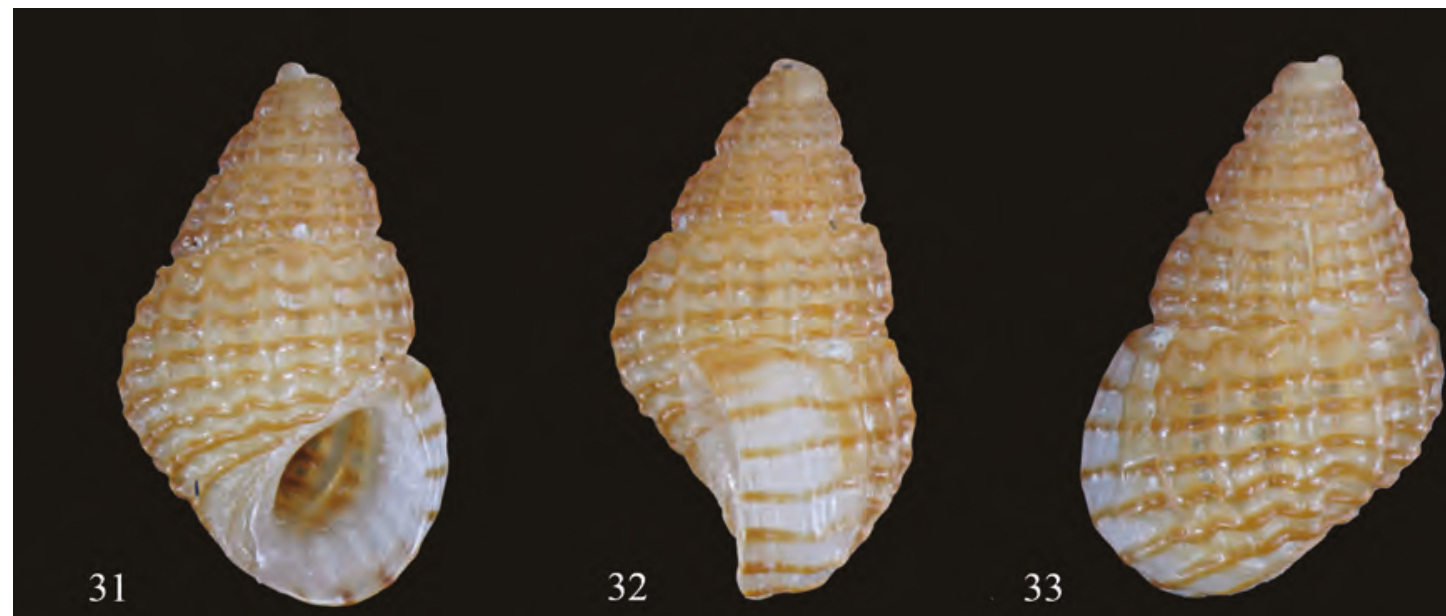

33
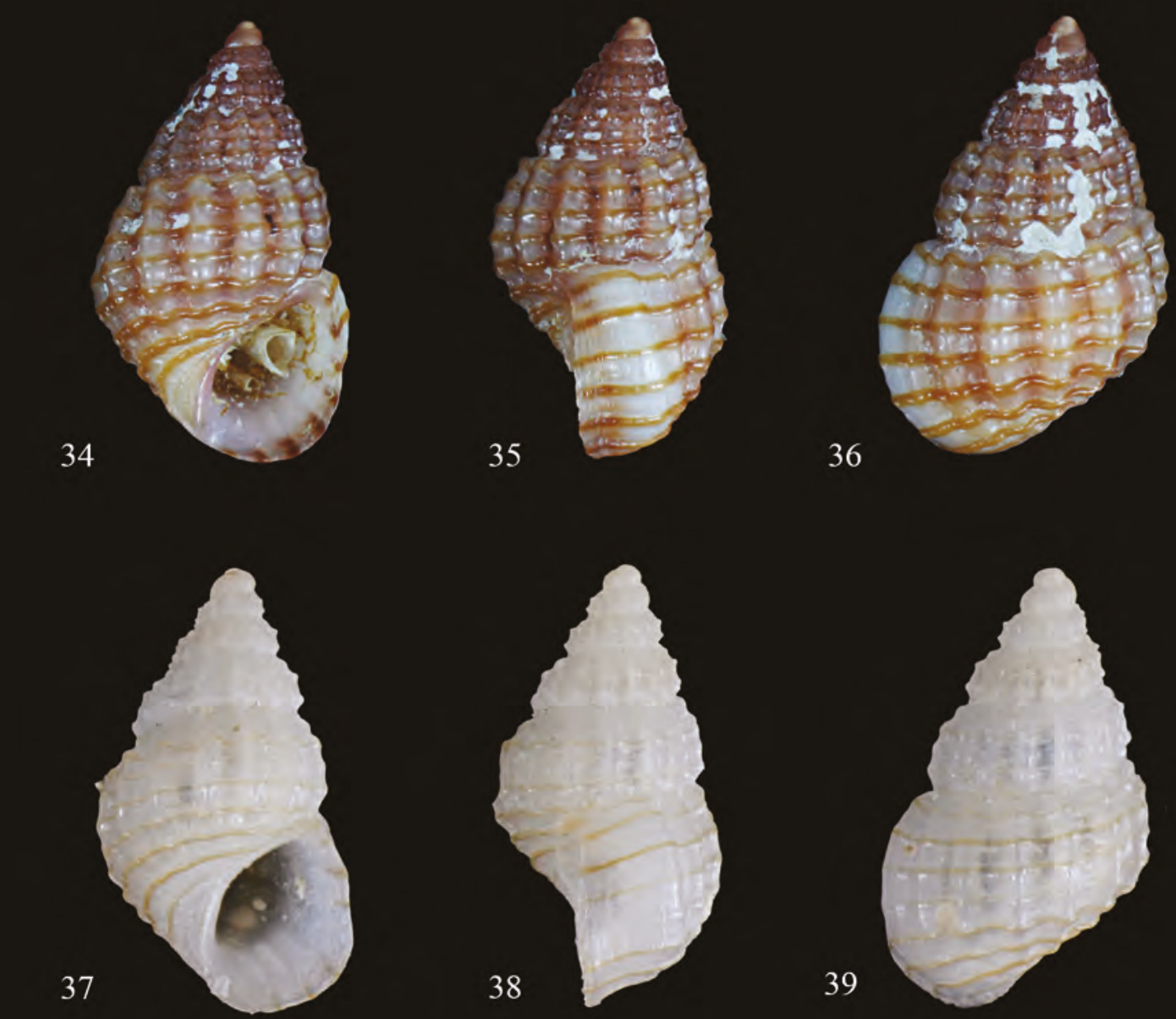

39

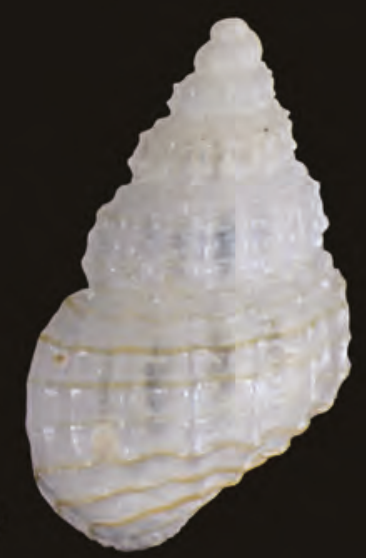

Figures 31-39. Alvania lineata Risso, 1826. Figs. 31-33: Gallipoli (Italy) $2.5 \mathrm{~m}$ depth, height $3.63 \mathrm{~mm}$ (DT). Figs. 34-36: Castro (Italy) $23 \mathrm{~m}$ depth, height $4.32 \mathrm{~mm}$ (DT). Figs. 37-39: Nardò, loc. S. Caterina (Italy) $23 \mathrm{~m}$ depth, height $3.15 \mathrm{~mm}$ (DT). 


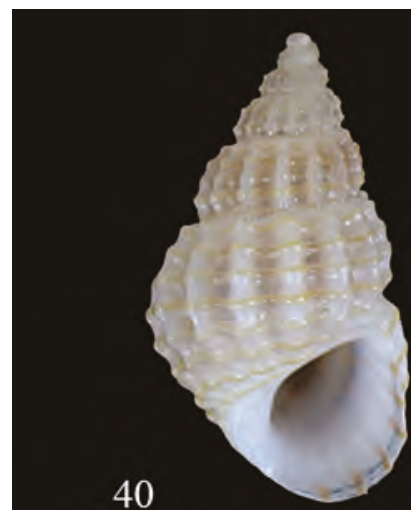

41

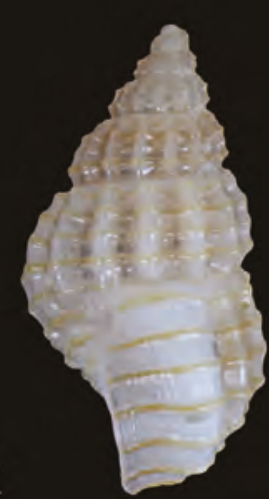

43

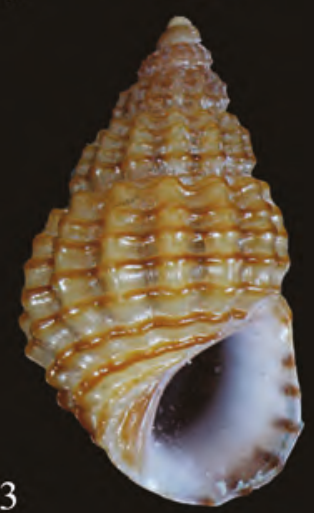

46

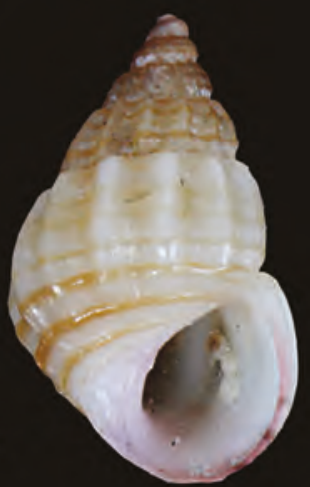

49

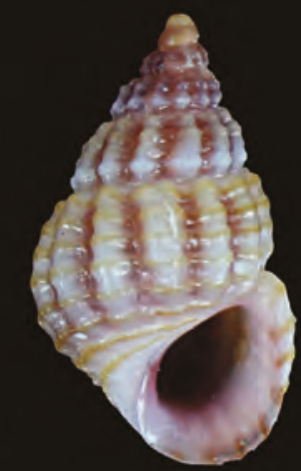

44

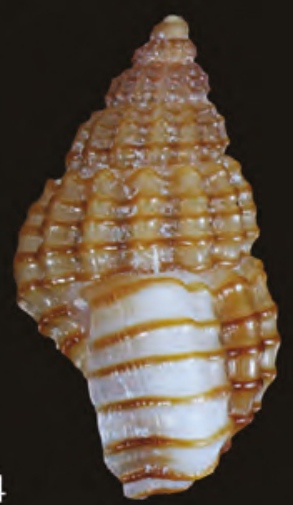

47

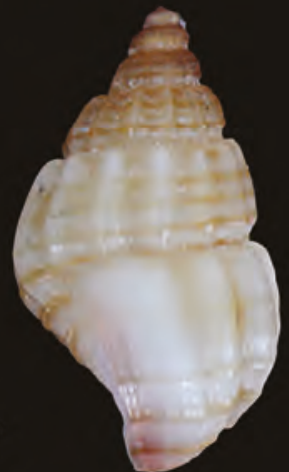

50

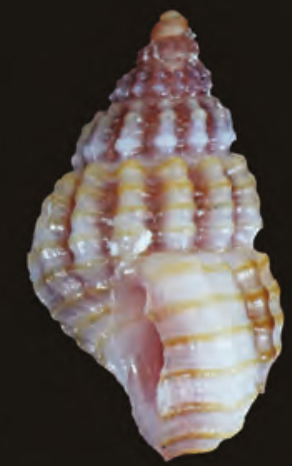

42

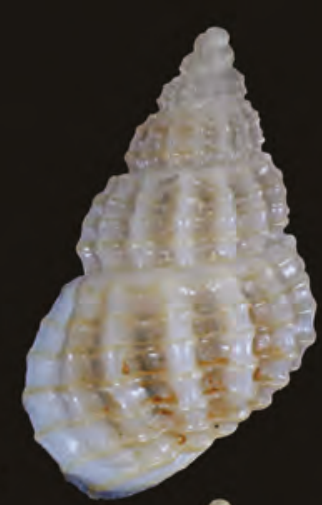

45

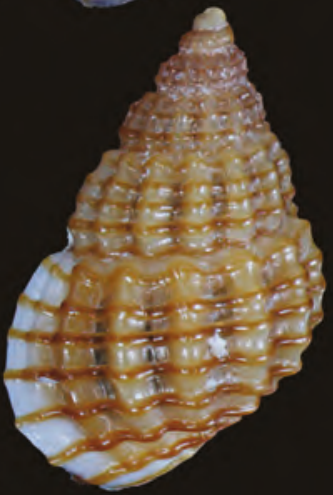

48

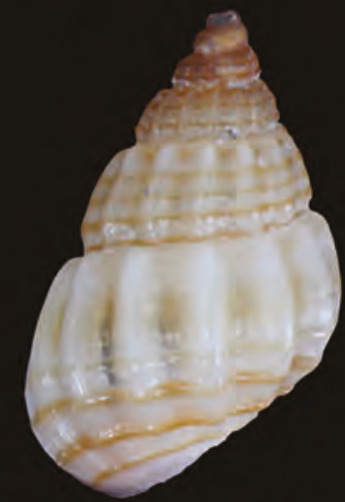

51

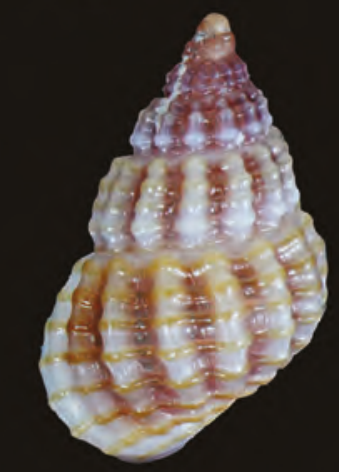

Figures 40-51. Alvania lineata Risso, 1826. Nardò (Lecce), località Torre Inserraglio (Italy) $23 \mathrm{~m}$ depth. Figs. 40-42: height $4.39 \mathrm{~mm}$ (DT). Figs. 43-45: height $4.23 \mathrm{~mm}$ (DT). Figs. 46-48: height $3.84 \mathrm{~mm}$ (DT). Figs. 49-51: height 3.91 $\mathrm{mm}(\mathrm{DT})$. 


\section{ACKNOWLEDGEMENTS}

The authors wish to thank Massimo Appolloni (MCZR, Roma, Italy) for his assistance during the visits to examine Monterosato's material; Angelo Vannozzi (Roma, Italy), Italo Nofroni (Roma, Italy), Antonio Flavio Tricarico (Gallipoli, Lecce, Italy), Pippi Guida (Copertino, Lecce, Italy), Angelo Fiorita (Porto Cesareo, Lecce, Italy), Walter Renda (Amantea, Cosenza, Italy), and Massimo Viva (Copertino, Lecce, Italy) for their friendly help, useful discussions, presented materials and photos.

\section{REFERENCES}

Amati B., 2012. Alvania consociella Monterosato, 1884 junior synonym of Alvania lanciae (Calcara, 1845) (Prosobranchia, Rissoidae). Bollettino Malacologico, 48: 116-121.

Amati B., 2014. Description of Alvania aliceae spec. nov. (Gastropoda, Rissoidae) from the Mediterranean Sea. Iberus, 32: 87-95.

Amati B. \& Smriglio C., 2016. Taxonomic notes on the Alvania dictyophora-complex with the description of Alvania desabatae spec. nov. (Gastropoda, Rissoidae) from the Mediterranean Sea. Iberus, 34: 163180.

Amati B., Nofroni I. \& Oliverio M., 1987. A revision of the Alvania rudis (Philippi, 1844) group (Gastropoda: Prosobranchia). La Conchiglia, 19 (216-217): 26-27.

Amati B., Appolloni M. \& Smriglio C., 2017. Taxonomic notes on the Alvania cimex-complex in the Mediterranean Sea. Alvania cingulata (Philippi, 1836) junior synonym of Alvania mamillata Risso, 1826 (Gastropoda, Rissoidae). Iberus, 35: 123-141.

Amati B., Appolloni M., Quaggiotto E., Smriglio C. \& Oliverio M., 2019. Notes on some taxa of the Alvania lineata-complex and descriptions of three new species of the genus Alvania Risso, 1826 from the Mediterranean Sea (Gastropoda: Rissoidae). Iberus, 37: 81-112.

Amati B., Appolloni M., Di Giulio A., Scuderi D., Smriglio C. \& Oliverio M., 2020. Revision of the Recent
Alvania scabra (Philippi, 1844) complex (Mollusca, Gastropoda, Rissoidae) from the Mediterranean Sea with the description of a new species. Zootaxa, 4767 (3): 415-458.

Bouchet P. \& Warén A., 1993. Revision of the Northeast Atlantic Bathyal and Abyssal Mesogastropoda. Bollettino Malacologico, Supplemento 3: 579-849.

Chiarelli S. \& Micali P., 2003. Vela Luka: lo Ionio in Adriatico. Notiziario S.I.M., 21: 27-30.

Gofas S. \& Oliver J. D. in Gofas S., Moreno D. \& Salas C., 2011. Moluscos marinos de Andalucía. Volumen I, pp. i-xvi y 1-342; Volumen II, pp. i-xii y 343-809. Málaga: Servicio de Publicaciones e Intercambio Científico, Universidad de Málaga.

Hadley A., 2006. Combine ZP public domain image processing software. Available from: https://web. archive.org/web/20160221032141/http://www.hadlyweb.pwp.blueyonder.co.uk/ (accessed 12 March 2020)

Micali P., Tisselli M. \& Giunchi L., 2005. Ritrovamenti malacologici alle isole Tremiti (Adriatico meridionale). Notiziario S.I.M., 22: 23-25.

MolluscaBase (Eds.), 2020. MolluscaBase. Alvania Risso, 1826. Accessed through: Worl Register of Marine Species at: http://www.marinespecies.org/aphia. php? $\mathrm{p}=$ taxdetails \&id $=138439$ on $2020-05-12$.

Nevill G., 1885. Hand list of Mollusca in the Indian Museum, Calcutta. Part II. Gastropoda, ProsobranchiaNeurobranchia (contd.). Government Printer, Calcutta, 306 pp.

Ponder W.F., 1985. A Review of the Genera of the Rissoidae (Mollusca: Mesogastropoda: Rissoacea). Records of the Australian Museum, Suplement 4 [1984], 1-221.

Scuderi D., 2014. A new species of rissoid of the genus Alvania Risso, 1826 from the E-Sicily: Alvania maximilicutiani n. sp. (Gastropoda Rissoidae). Biodiversity Journal, 5: 201-208.

Scuderi D. \& Terlizzi A., 2012. Manuale di malacologia dell'Alto Ionio. Edizioni Grifo, Manduria, 186 pp.

Tringali L.P., 2001. Marine malacological records (Gastropoda: Prosobranchia, Heterobranchia, Opisthobranchia and Pulmonata) from Torres de Alcala, Mediterranean Morocco, with the description of a new philinid species. Bollettino Malacologico, 37 : 207-222. 
